\title{
Confined linear carbon chains as a route to bulk carbyne
}

\author{
Lei Shi', Philip Rohringer', Kazu Suenaga ${ }^{2}$, Yoshiko Niimi' ${ }^{2}$, Jani Kotakoski', Jannik C. Meyer', \\ Herwig Peterlik, ${ }^{1}$ Marius Wanko ${ }^{3}$, Seymur Cahangirov ${ }^{3,4}$, Angel Rubio ${ }^{3,5}$, Zachary J. Lapin ${ }^{6}$, \\ Lukas Novotny ${ }^{6}$, Paola Ayala ${ }^{1,7}$ and Thomas Pichler ${ }^{1 \star}$
}

Strong chemical activity and extreme instability in ambient conditions characterize carbyne, an infinite $\boldsymbol{s p}^{1}$ hybridized carbon chain. As a result, much less has been explored about carbyne as compared to other carbon allotropes such as fullerenes, nanotubes and graphene. Although end-capping groups can be used to stabilize carbon chains, length limitations are still a barrier for production, and even more so for application. We report a method for the bulk production of long acetylenic linear carbon chains protected by thin double-walled carbon nanotubes. The synthesis of very long arrangements is confirmed by a combination of transmission electron microscopy, $X$-ray diffraction and (near-field) resonance Raman spectroscopy. Our results establish a route for the bulk production of exceptionally long and stable chains composed of more than 6,000 carbon atoms, representing an elegant forerunner towards the final goal of carbyne's bulk production.

$\mathrm{D}$ ifferent kinds of allotropes can be formed from elemental carbon owing to its $s p^{n}$ hybridization ${ }^{1}$. Well known are diamond and tetrahedral amorphous carbon as $s p^{3}$ hybridized solids, whereas $s p^{2}$ hybridization is present in graphite, fullerenes ${ }^{2}$, carbon nanotubes (CNTs; ref. 3) and graphene $e^{4}$. All of them have been extensively investigated in recent decades. Carbyne, an infinite carbon chain with $s p^{1}$ hybridization, has attracted much interest and significant controversy ${ }^{5-8}$ since the 1960s. Its identification remained questionable for years ${ }^{9,10}$ until the production of polyynes: short $s p^{1}$ hybridized linear carbon arrangements with end-capping groups. The longest reported polyynes so far consist of 44 contiguous carbon atoms with alternating single and triple bonds $^{11}$, but the bulk synthesis of very long linear carbon chains (LLCCs) continues to face stumbling blocks. Little is known about the $s p^{1}$ hybridization in carbyne because of its extreme instability in ambient conditions. In fact, a longstanding study postulated the impossibility of preparing this truly one-dimensional (1D) material $^{12}$. The same had been foreseen for graphene on account of its thermodynamic instability, but the recent reports of feasible synthesis routes have inspired revisiting carbyne from both theoretical and experimental points of view. Carbyne's theoretically anticipated strength, elastic modulus and stiffness are greater than any known material, including diamond, carbon nanotubes and graphene, and allows one to envisage new composite materials ${ }^{13}$. Other essentially unexplored applications involve reaching the utmost limit in channel width for field-effect transistors (one-carbonatom thickness) ${ }^{14}$. The experimental research in this field has focused heavily on the synthesis of linear carbon chains (LCCs) by different methods, among which, heavy end-capping groups have commonly been used for stabilization ${ }^{11,15,16}$. At the same time, single(SW), double-(DW) and multiwalled (MW)CNTs have increasingly been used as confining nanoreactors where novel one-dimensional materials can be produced, such as short polyynes ${ }^{17}$, metal nanowires $^{18}$ and ultra-narrow graphene ${ }^{19}$. MWCNTs have been reported as hosts of carbon nanowires with approximately 100 atoms using direct arc discharge ${ }^{20}$. DWCNTs have facilitated the growth of short carbon chains from high-temperature treatments of buckypaper ${ }^{21}$, as well as carbon nanowires, by promoting the fusion of molecules such as $\mathrm{C}_{10} \mathrm{H}_{2}$ or adamantane ${ }^{22,23}$. Here, we have sought to use the full potential of narrow-diameter DWCNTs both as nanoreactors and as a tool to encapsulate and protect LLCCs. Our procedure uses very high temperature and high vacuum (HTHV), allowing the bulk realization of the longest LLCCs ever observed, consisting of more than 6,000 contiguous acetylenic carbons inside DWCNTs with ideal diameters. The high recurrence of LLCCs inside DWCNTs (LLCCs@DWCNTs) substantiate a truly feasible path towards the bulk-scale formation of carbyne.

Direct observations of the LLCCs by aberration-corrected high-resolution transmission electron microscopy (HRTEM) and scanning transmission electron microscopy (STEM) are summarized in Fig. 1. The HRTEM micrograph in Fig. 1a shows a long, bent DWCNT encapsulating a carbon chain. Taking into account that the single- and triple-bond lengths are 0.1329 and $0.1229 \mathrm{~nm}$, exhibiting a calculated lattice constant of $d=0.2558 \mathrm{~nm}$, more than 200 contiguous carbon atoms are contained within the $\sim 26 \mathrm{~nm}$ shown in Fig. 1a. This perfectly matches our complementary bulk-scale X-ray diffraction results revealing a lattice constant of $d=0.252 \mathrm{~nm}$ and an average length of $40 \mathrm{~nm}$ (more than 300 carbon atoms; see Supplementary Fig. 21). To the best of our knowledge, the LLCC in Fig. 1a is the longest directly observed in TEM. These microscopy studies also confirm tubes can be partially filled (Fig. 1b). Normalizing the XRD spectra

\footnotetext{
${ }^{1}$ University of Vienna, Faculty of Physics, 1090 Wien, Austria. ${ }^{2}$ National Institute of Advanced Industrial Science and Technology (AIST), Nanotube Research Centre, 305-8565 Tsukuba, Japan. ${ }^{3}$ Nano-Bio Spectroscopy Group and European Theoretical Spectroscopy Facility (ETSF), Universidad del País Vasco, CFM CSIC-UPV/EHU-MPC\&DIPC, 20018 San Sebastián, Spain. ${ }^{4}$ UNAM-National Nanotechnology Research Center, Bilkent University, 06800 Ankara, Turkey. ${ }^{5}$ Max Planck Institute for the Structure and Dynamics of Matter, 22761 Hamburg, Germany. ${ }^{6}$ ETH Zürich, Photonics Laboratory, 8093 Zürich, Switzerland. ${ }^{7}$ Yachay Tech University, School of Physical Sciences and Nanotechnology, 100119-Urcuquí, Ecuador. 


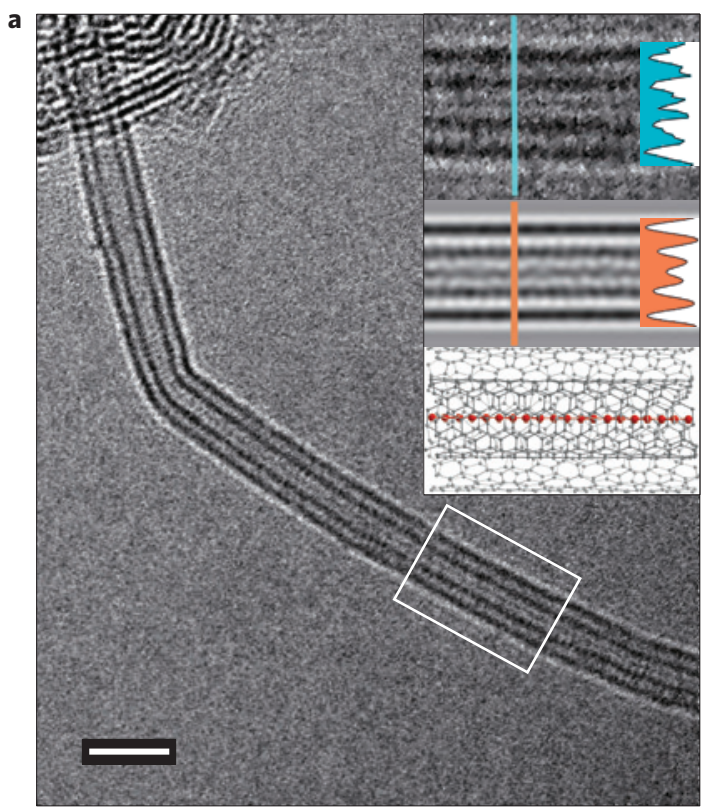

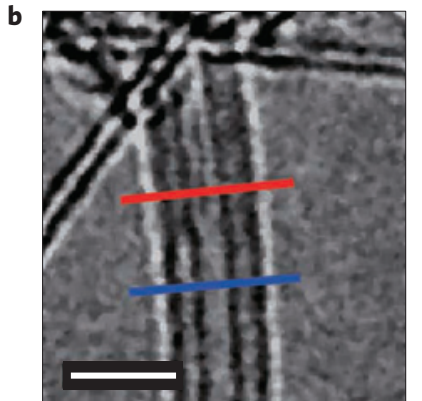

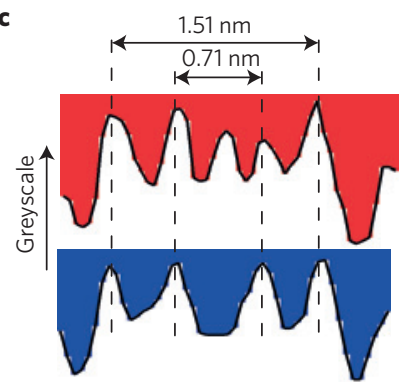

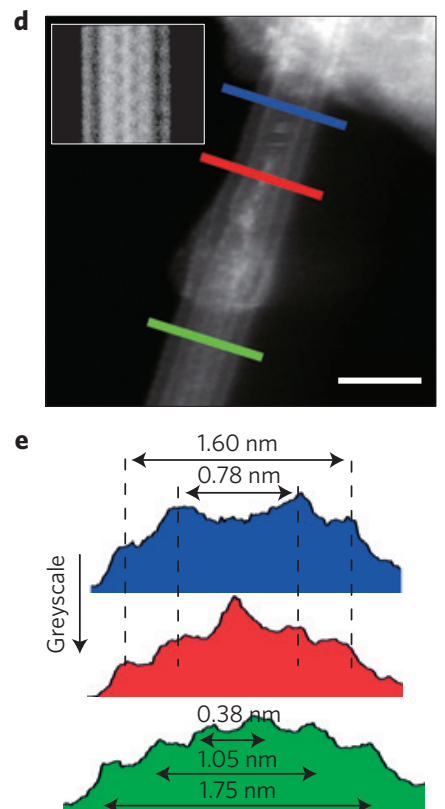

Figure 1 | Direct observation of LLCCs@DWCNTs. a, HRTEM image of an LLCC@DWCNT with bending. The LLCC inside a DWCNT is longer than 26 nm, which means that it consists of more than 200 contiguous carbon atoms. Inset: an enlarged part of the HRTEM image (top), a simulated HRTEM of an LLCC@DWCNT (middle) and a molecular model of an LLCC@DWCNT (bottom). The corresponding line profiles of the experimental and a simulated LLCC@DWCNT are shown, respectively. b, A DWCNT with partial LLCC filling. c, The line profiles at positions along the blue and red lines shown in b represent the empty DWCNT and LLCC@DWCNT, respectively.d, STEM image of an LLCC@DWCNT. Inset: a simulated STEM image of an LLCC@DWCNT. e, The line profiles at positions along the blue, red and green lines shown in d represent an empty DWCNT, an LLCC@DWCNT and a thin most-inner tube@DWCNT, respectively. Scale bars, 2 nm.

with known samples, combined with the Raman results presented below, support that more than $90 \%$ of DWCNTs with inner tube diameters between $0.62-0.85 \mathrm{~nm}$ can be filled with LLCCs (for details see Supplementary Section 7).

HRTEM simulations were also used to confirm LLCCs (inset of Fig. 1a and Supplementary Figs 9 and 10). Comparing empty and filled parts of a DWCNT (Fig. 1c), a peak corresponding to the LLCC inside a DWCNT is observed. Complementary STEM studies have been performed, as shown in Fig. 1d, and are in good agreement with the HRTEM results (see simulations in Supplementary Fig. 11 and line profiles in Fig. 1e). The green profile (Fig. 1e) corresponds to a triple-walled nanotube. This allows us to propose that the HTHV nanochemical reactions form LLCCs only for DWCNTs with outer diameters below $\sim 1.75 \mathrm{~nm}$. For slightly thicker DWCNTs, triplewalled CNTs are formed and significantly larger diameter tubes host twisted carbon clusters (Supplementary Fig. 7). This establishes the diameter as a key parameter for the growth of LLCCs. Although HRTEM and STEM are extremely helpful, the fine structure of the LLCCs remains elusive owing to the movement of the LLCCs inside the DWCNTs.

To further understand the effect of confinement on the growth of LLCCs, we performed density functional theory (DFT) calculations with the Vienna $A b$ Initio simulation package (VASP; refs 24,25) using projector augmented wave potentials ${ }^{26}$ for chains inside four small-diameter armchair nanotubes, namely, $(4,4),(5,5),(6,6)$ and $(7,7)$, with one repeating unit in the length direction. The most stable chain configuration is exactly straight, with alternating single and triple bonds, with bond lengths of $1.329 \AA$ and $1.229 \AA$, respectively. The interaction energies between the carbon chains and the different nanotubes are plotted in Fig. 2, and the inset shows illustrations of the LLCC inside different CNTs. The results show that the optimum distance between the carbon chain and the CNT is $0.3378 \mathrm{~nm}$, which is very close to the optimum inter-layer distance between graphite layers $(\sim 0.32 \mathrm{~nm})$, as predicted by the DFT-D2 $\operatorname{method}^{27}$. The LLCC@ $(5,5)$ exhibits the lowest interaction energy,

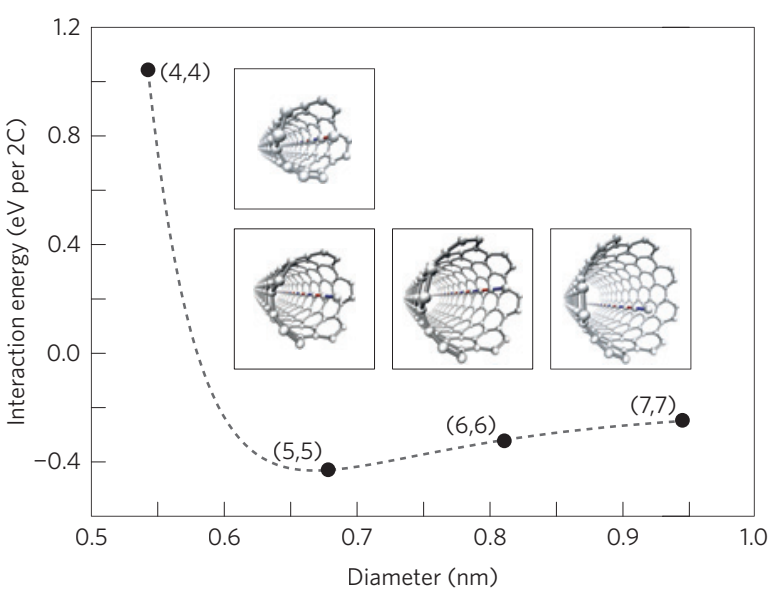

Figure 2 | Calculation of the optimum CNT diameter for LLCC encapsulation. DFT calculations for the interaction energies between the LLCC and different chirality tubes: $(4,4),(5,5),(6,6)$ and $(7,7)$. Inset: illustrations of different LLCC@CNT structures. Notice that the distance between the LLCC and the wall of the CNT remains similar for all host CNTs. For the energy minimum, this distance is close to the radius of the CNT.

which means that a $(5,5)$ nanotube with a diameter of $0.69 \mathrm{~nm}$ is the optimum among the four different proposed CNTs for LLCC growth. Assuming that the scaling between the theoretical and experimental values of the graphite inter-layer spacing is the same as that for the spacing between carbyne and the CNT, the predicted optimum tube diameter is $\sim 0.71 \mathrm{~nm}$, in very good agreement with our experiments.

Resonance Raman spectroscopy was used to verify the optimal DWCNT diameter and growth conditions for the bulk-yield synthesis of LLCCs. The LLCCs exhibit a Raman active mode named the LLCC-band ${ }^{20,22,28}$. Polyynes with fewer than 20 carbon 

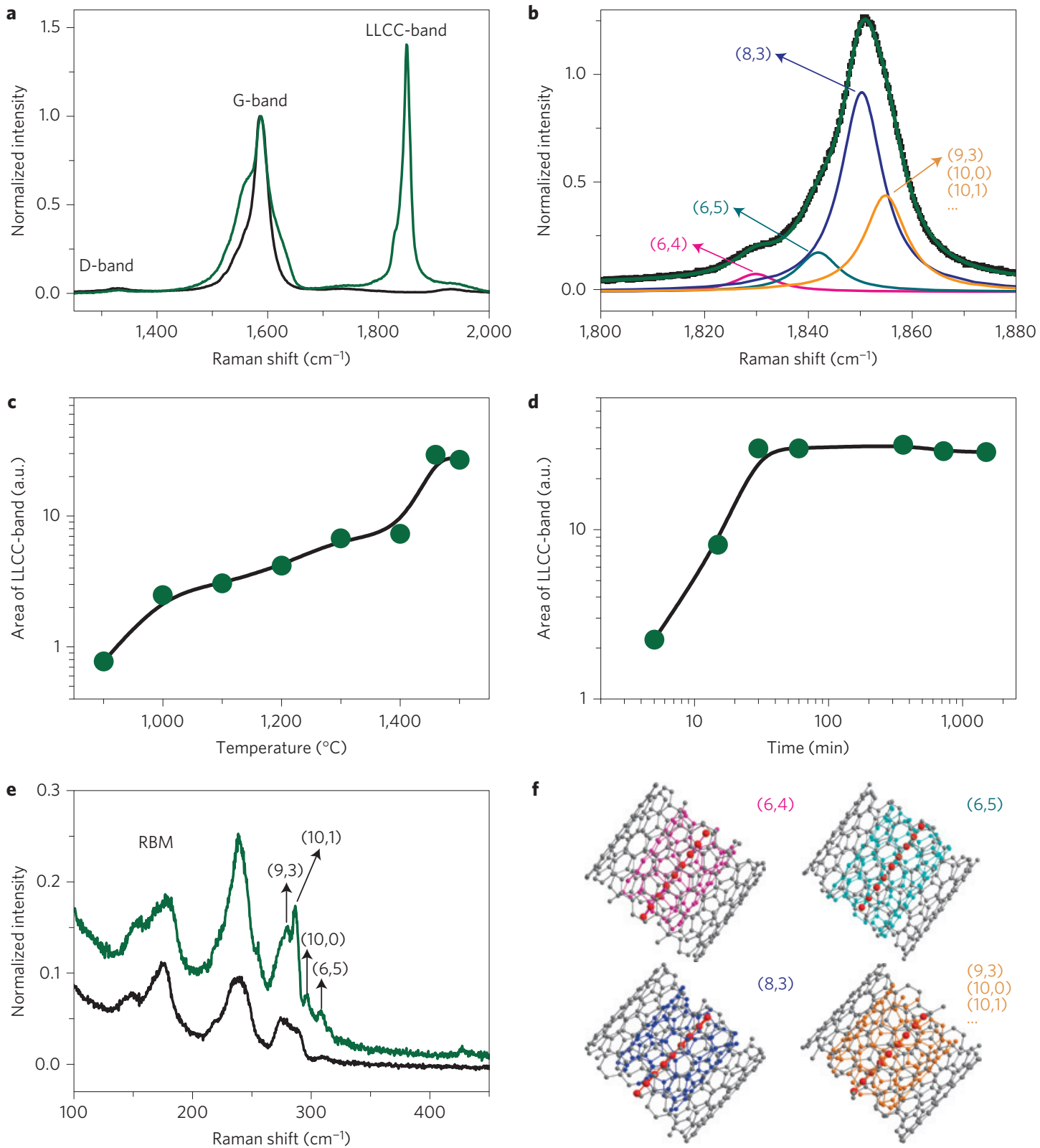

$\mathbf{f}$

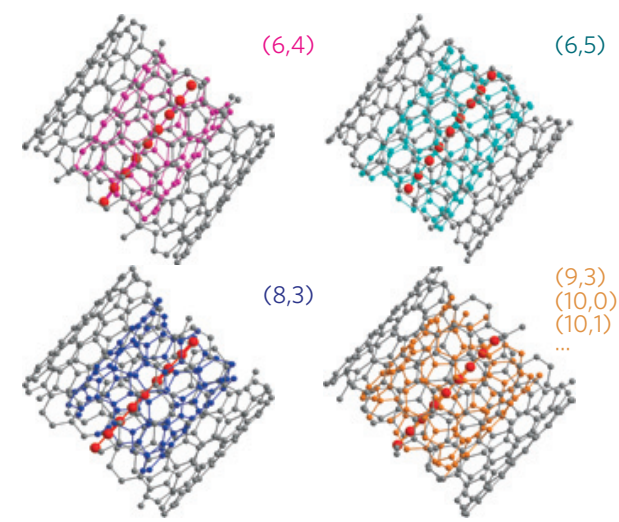

Figure 3 | Raman spectra of LLCCs@DWCNTs carried out at an excitation wavelength of $\mathbf{5 6 8 . 2} \mathbf{n m}$. a, D-band, G-band and LLCC-band (green line) of a sample annealed at $1,460^{\circ} \mathrm{C}$ as compared to pristine DWCNTs (black line). b, LLCC-band line shape analysis includes four components. The green line shows their resulting envelope, the black dotted line represents the original data. c, Area of the LLCC-band as a function of annealing temperature for 30 min annealing time. d, Area of the LLCC-band as a function of annealing time at $1,460^{\circ} \mathrm{C}$. e, RBM region for a sample annealed at $1,460^{\circ} \mathrm{C}$ ( $\mathrm{green}$ line) as compared to pristine DWCNTs (black line). f, Schemes of our most abundant inner tube chiralities and chain configurations.

atoms have bands between 1,900 and $2,300 \mathrm{~cm}^{-1}$ (ref. 29), whereas longer chains of about 100 atoms in MWCNTs have a response between $\sim 1,825$ and $1,850 \mathrm{~cm}^{-1}$ (ref. 20). For LLCCs@DWCNTs, we observe that the DWCNTs are not damaged when the LLCCs are formed, as confirmed by the same ratio between the defect-induced (D-band) and graphitic mode (G-band) of pristine DWCNTs and annealed DWCNTs containing LLCCs (see Fig. 3a). Moreover, after annealing, a very intense LLCC-band with a fine structure is observed at about $\sim 1,850 \mathrm{~cm}^{-1}$. A detailed line shape analysis is shown in Fig. 3b. The LLCC-band was always found between 1,780 and $1,880 \mathrm{~cm}^{-1}$, concomitant with an intensity several times larger than in any previous works on polyynes. Complementary multi-frequency studies for these LLCCs@DWCNTs confirm a broad resonance window of the LLCC-band, peaking at about $568 \mathrm{~nm}$ (see Supplementary Information).
The low energy of the Raman mode confirms that the chains are always very long and the high mode intensity (relative to G-band) suggests that production is feasible in bulk quantities. Although we can not directly determine the LLCC growth yield, we show that it depends strongly on the growth time and temperature in high vacuum $(\mathrm{HV})$. Figure $3 \mathrm{c}$ summarizes the changes in the area of the LLCC-band as a function of annealing temperature. Our HTHV method allows the synthesis of a small quantity of LLCCs at temperatures as low as $900{ }^{\circ} \mathrm{C}$. This is consistent with reports on the fusion of linear polyynes or adamantane molecules inside DWCNTs at similar temperatures, but those linear structures grow in low yields that depend on their initial filling ratio $^{22,23}$. The integrated LLCC-band intensity peaks at an annealing temperature of $1,460{ }^{\circ} \mathrm{C}$. Annealing at even higher temperatures leads to a subsequent decomposition of the LLCC@DWCNT 

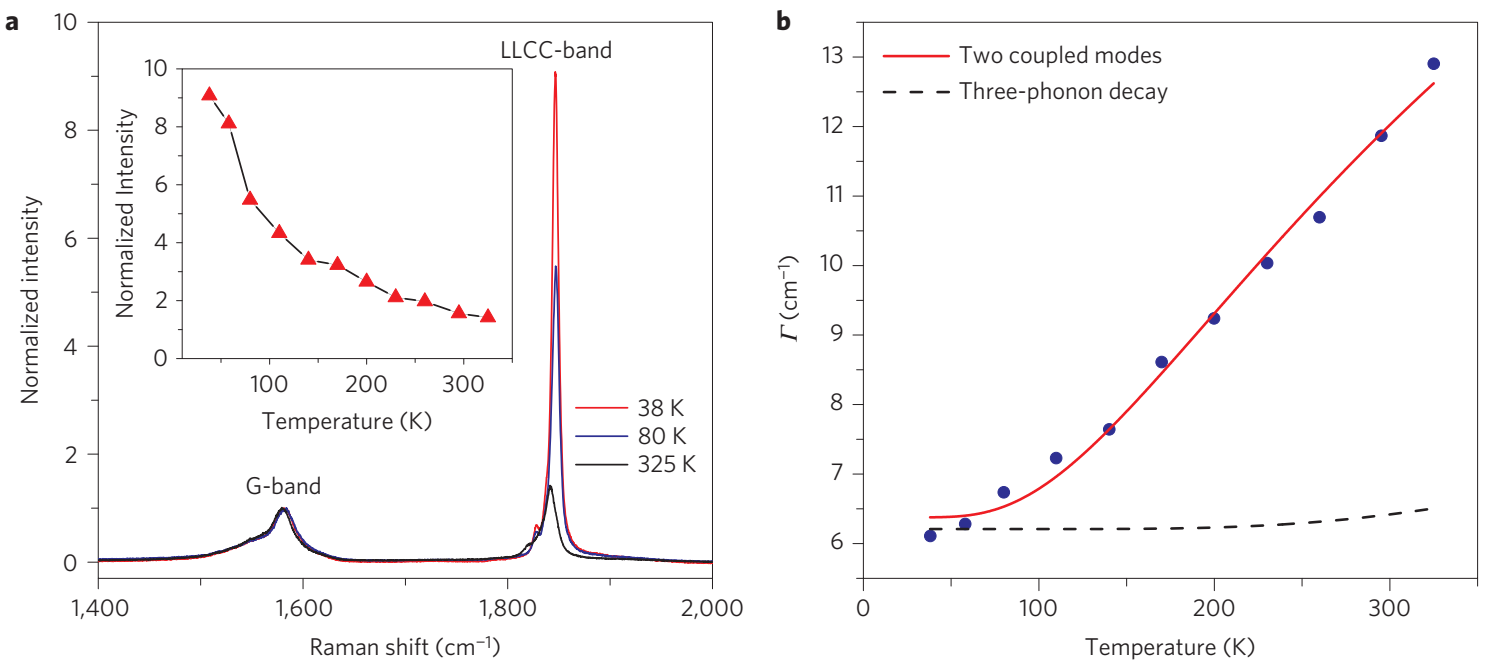

Figure 4 | The temperature dependence of the LLCC-band. a, Raman spectra of LLCCs@DWCNTs measured at different temperatures. The spectra are normalized to the G-band response. The inset shows the LLCC-band intensity in comparison to the G-band. $\mathbf{b}$, FWHM of the individual components in the LLCC-band as a function of temperature (blue circles). The solid line represents a model of a temperature-dependent coupling among different modes, the dashed curve represents a fit to a model of a three-phonon decay.

structure. Aiming at understanding the growth kinetics of the LLCCs, the pristine DWCNTs were also annealed in HV at the optimal temperature of $1,460^{\circ} \mathrm{C}$ for different durations. As observed in Fig. 3d, the overall LLCC-band appears after five minutes of annealing, and increases for $25 \mathrm{~min}$ before saturating, suggesting that the LLCC growth is complete. However, the longer chains still continue to grow, as revealed by a line shape analysis of the LLCCs showing that the relative intensity of lower-frequency LLCC-band peaks continue to increase. The key to the successful growth of long LLCCs is the ability to anneal small-diameter CNTs (protected by an outer CNT) in HV, facilitating a stable host CNT for the growth of the LLCC. SWCNTs with a diameter of less than $\sim 0.7 \mathrm{~nm}$ are not stable at high temperature, and therefore very few LLCCs can be grown inside them (see Supplementary Information).

A remaining important question is how the LLCC structure is confined inside the tubes. This missing piece of the puzzle can be retrieved from an analysis of the radial breathing modes (RBMs) of the DWCNTs, depicted in Fig. 3e. DWCNTs can be indexed by their chiralities using the Kataura plot, including a correction term for the inter-tube distance ${ }^{30,31}$. The indexed RBM peaks corresponding to the thin inner tubes are split and enhanced. This is a first indication of an interaction with the LLCCs. Thinner host tubes interact more strongly with the LLCC, and therefore a lower-frequency Raman response is observed. This enables us on the other hand to tentatively assign the bulk LLCC line shape fine structure (Fig. 3b) to different chiralities of inner host tubes. Considering the total energy of the LLCC@DWCNT, $(6,4)$ tubes are more prone to host longer LLCCs than $(6,5)$ tubes. At the same time, a $(6,4)$ tube has a larger interaction with its encapsulated LLCC than a $(6,5)$ tube. Further exploring the fine structure of the LLCC-band allows not only an estimation of the growth yield of different LLCCs@DWCNTs through the different relative intensities of the LLCC-bands, but also an easy comparison of the growth yield of LLCCs between different samples. This analysis relies on a weak van der Waals (vdW) coupling between the inner tubes and the LLCCs, which is confirmed by analysing the temperaturedependent Raman response of the LLCCs. The intensity and area of the LLCC-band are both much greater at low temperatures, as shown in Fig. 4a. The data recorded from 325 to $38 \mathrm{~K}$, plotted in the inset, show the LLCC-band intensity increasing from 156\% of the G-band intensity at room temperature to $908 \%$ of the G-band intensity at $38 \mathrm{~K}$. Concomitantly, it was observed that the full-width at half-maximum (FWHM, $\Gamma$ ) is noticeably reduced (Fig. 4b). Normally, the temperature dependence of the FWHM can be analysed by a classical model of anharmonic decay of optical phonons $^{32-34}$ or a model of a temperature-dependent coupling among different modes. As shown in Fig. 4b, the anharmonic decay does not fit our experimental results. Hence, we describe our results by the second model, that is, a coupling of the LLCC-band to the RBM of the inner tube. The temperature dependence of the FWHM follows the following equation, with an activation energy $E_{\mathrm{a}}$ (ref. 35).

$$
\Gamma(T)=\Gamma(0)\left(1+\mathrm{e}^{-E_{\mathrm{a}} / k_{\mathrm{B}} T}\right)
$$

Using an activation energy of $E_{\mathrm{a}}=33.85 \mathrm{meV}$, we find an excellent agreement with our experimental results, as shown by the solid line in Fig. 4b. This strongly supports the coupling of the LLCCs to the inner tubes of the DWCNTs, and highlights the importance of the confined nanospace with the right diameter, as introduced above. This activation energy is also consistent with a weak vdW interaction between the tubes and the LLCCs.

Building further on these findings, we can analyse the relative Raman shift as a function of the inverse chain length. This has been performed in previous studies by comparing to theory using an empirical bond length alternation model which was originally introduced for polyacetylen $\mathrm{e}^{36}$. These previous theoretical calculations were performed for polyynes in vacuo ${ }^{37-39}$, and agree well with our new spin-component-scaled (SCS) MP2 ab initio calculations for chains in vacuo (see Supplementary Fig. 22). All of these calculations of freestanding chains have found that the Raman shift saturates for infinitely long chains.

To analyse the Raman response with the length of very long LLCCs, we measured several individual LLCCs@DWCNTs by nearfield Raman spectroscopy (for more details see Supplementary Section 8). A typical atomic force microscopy (AFM) image including two individual tubes is depicted in Fig. 5a. The Raman spectrum of these two individual LLCC@DWCNTs include only one respective narrow Lorentzian peak at Raman frequencies of $1,796 \pm 1$ and $1,800 \pm 1 \mathrm{~cm}^{-1}$ (see Fig. 5d). Their particular measured lengths are 290 and $190 \mathrm{~nm}$, as derived from the intensity map of the LLCCs (depicted in the colour overlay of Fig. 5a,b). The longest observed chain had a length of $797 \mathrm{~nm}$ and a corresponding Raman frequency of $1,791 \pm 1 \mathrm{~cm}^{-1}$, as shown in Fig. $5 \mathrm{e}, \mathrm{f}$. The three LLCCs represent chain lengths between approximately 1,500 and 

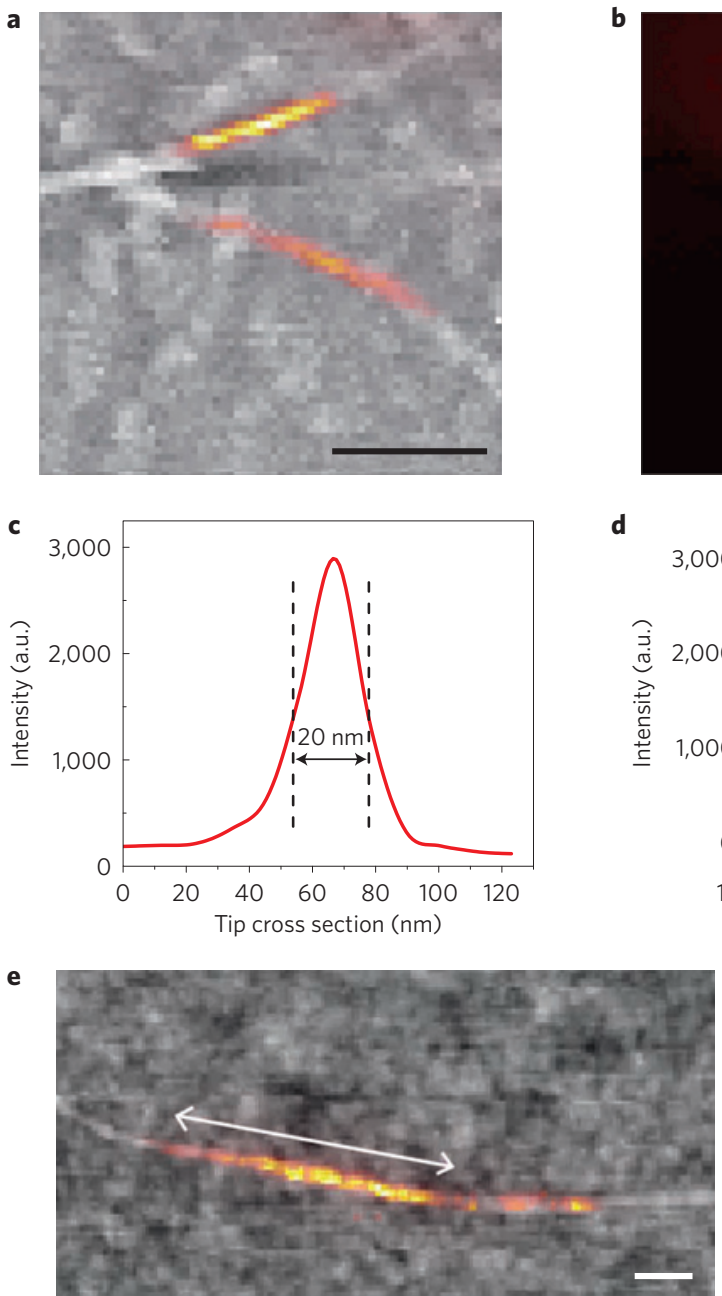

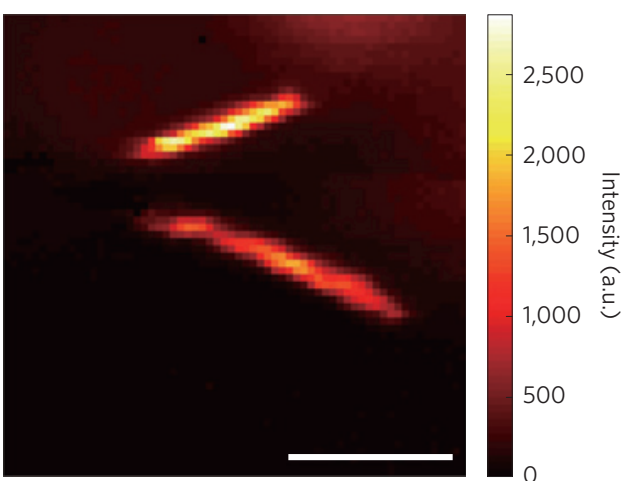

d
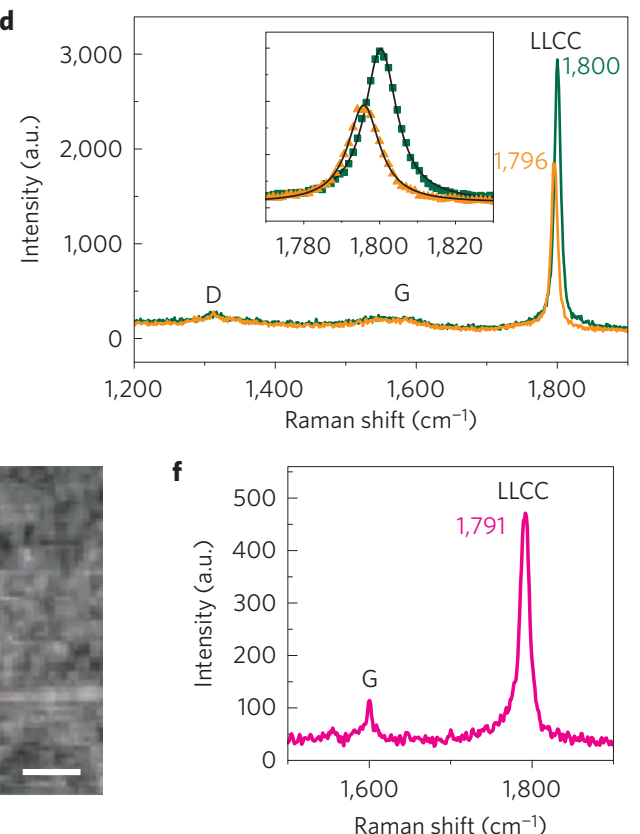

Figure 5 | Comparison of the inverse length of linear carbon chains with the Raman response. a, Overlay of the near-field Raman signal of the LLCC-band (colour) on the topographic AFM Image (greyscale). b, Raman mapping of two individual LLCCs@DWCNTs by signal filtering: 1,796 and 1,800 cm-1, corresponding to the top and bottom tubes, respectively. c, Optical cross section for the near-field probe. d, Raman spectra of the top and bottom LLCC@DWCNT using 647.1 nm excitation. The inset shows the fitting of the LLCC-bands by a single Lorentzian peak (black solid lines) in an extended

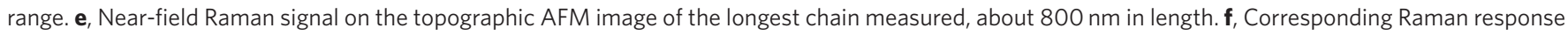
of this ultra-long chain at $1,791 \mathrm{~cm}^{-1}$. All scale bars are $200 \mathrm{~nm}$.

more than 6,000 carbon atoms. Together with two more chains of $320 \mathrm{~nm}$ and $505 \mathrm{~nm}$, the observed spread in the Raman frequency of ultra-long LLCCs is $17 \mathrm{~cm}^{-1}$ (that is, between $1,787 \mathrm{~cm}^{-1}$ and $1,804 \mathrm{~cm}^{-1}$ with an uncertainty in the line position of $\pm 1 \mathrm{~cm}^{-1}$ due to near-field interactions). It remains inconclusive if the Raman frequency of ultra-long chains saturates with chain length, as reported by the existing theory of chains in vacuum, or if additional effects of the nanotube environment need to be taken into account (ongoing work).

Although the exact mechanism of the LLCC@DWCNT formation through HTHV nanochemical reactions remains unknown, our in-depth inspection of the material allows us to suggest a model based on the confinement inside the circular nanospace of the tubes. Considering the lowest energy principle, carbon atoms can move inside the nanotubes from the open ends or through the tube walls during the $\mathrm{HV}$ annealing process. The tubes with diameter of $\sim 0.71 \mathrm{~nm}$ are best suited to grow LLCCs, as slightly larger tubes tend to form spiral chains or, if the tube diameter is too large, they no longer work as nanoreactors and the LLCCs disintegrate. With our narrow diameter distribution of inner tubes we are able to achieve a bulk production of LLCCs.
Annealing a mono-disperse sample of DWCNTs of an appropriate chirality, along with an additional carbon supply, may be a route to achieve the growth of bulk carbyne, which may then be extracted and stabilized in different environments.

Hence, we have developed a route for truly bulk production of LLCCs - that is, carbyne-confined inside DWCNTs. We find that the stability of the LLCCs depends crucially on spatial confinement, resulting in a coupling between the chains and the DWCNT host. For long chains, and small inner tubes, this also represents an interaction between the tubes and the chains. This suggests a possibility to directly correlate the LLCC length to the diameter of the inner tubes and determine the optimal diameter for the highyield growth of carbyne. Our longest LLCCs can be correlated to a chain length of more than 6,000 carbon atoms, which can be seen as the closest realization of carbyne so far.

The LLCCs grown with a high yield inside of DWCNTs are very stable, which is of great importance for further applications. Theoretical studies have shown that, after inserting LLCCs inside CNTs, the LLCC@DWCNT hybrid system would show metallic character due to charge transfer from CNTs to the LLCCs, despite the fact that both the CNTs and LLCCs have semiconductor 
properties themselves ${ }^{40,41}$. Therefore, we suggest that it is possible to control the electronic properties of the hybrid system by varying the filling yield of LLCCs. Furthermore, quantum spin transport in LLCCs is a promising theoretically predicted application ${ }^{42}$. As a true 1D nanocarbon, this novel LLCC@DWCNT system could be a candidate for nanoelectronic devices, in addition to its general appeal in physics and chemistry. It may also be possible to extract the LLCCs from their DWCNT host and stabilize them in a liquid environment for further applications.

\section{Methods}

Methods and any associated references are available in the online version of the paper.

\section{Received 25 September 2014; accepted 2 March 2016; published online 4 April 2016}

\section{References}

1. Hirsch, A. The era of carbon allotropes. Nature Mater. 9, 868-871 (2010)

2. Kroto, H. W., Heath, J. R., Obrien, S. C., Curl, R. F. \& Smalley, R. E. $\mathrm{C}_{60}$ : buckminsterfullerene. Nature 318, 162-163 (1985).

3. Iijima, S. Helical microtubules of graphitic carbon. Nature 354, 56-58 (1991).

4. Novoselov, K. S. et al. Electric field effect in atomically thin carbon films. Science 306, 666-669 (2004).

5. Kasatochkin, V. I., Sladkov, A. M., Kudryavtsev, Y. P., Popov, N. M. \& Korshak, V. V. Crystalline forms of a linear modification of carbon. Dokl. Akad. Nauk SSSR 177, 358-360 (1967).

6. Goresy, A. E. \& Donnay, G. A new allotropic form of carbon from Ries Crater. Science 161, 363-364 (1968).

7. Whittaker, A. G. \& Kintner, P. L. Carbon: observations of new allotropic form. Science 165, 589-591 (1969).

8. Sladkov, A. \& Kudryavtsev, Y. Diamond, graphite, carbyne: allotropic forms of carbon. Priroda 5, 37-44 (1969)

9. Smith, P. P. K. \& Buseck, P. R. Graphitic carbon in the Allende meteorite: a microstructural study. Science 212, 322-324 (1981).

10. Smith, P. P. K. \& Buseck, P. R. Carbyne forms of carbon: do they exist? Science 216, 984-986 (1982).

11. Chalifoux, W. A. \& Tykwinski, R. R. Synthesis of polyynes to model the sp-carbon allotrope carbyne. Nature Chem. 2, 967-971 (2010).

12. Baeyer, A. Ueber polyacetylenverbindungen. Ber. Dtsch. Chem. Ges. 18, 2269-2281 (1885).

13. Liu, M. J., Artyukhov, V. I., Lee, H., Xu, F. B. \& Yakobson, B. I. Carbyne from first principles: chain of $\mathrm{C}$ atoms, a nanorod or a nanorope. ACS Nano 7, 10075-10082 (2013).

14. Baughman, R. H. Dangerously seeking linear carbon. Science 312, 1009-1010 (2006).

15. Johnson, T. \& Walton, D. Silylation as a protective method in acetylene chemistry: polyyne chain extensions using the reagents, $\mathrm{Et}_{3} \mathrm{Si}(\mathrm{C} \equiv \mathrm{C})_{m} \mathrm{H}$ $(m=1,2,4)$ in mixed oxidative couplings. Tetrahedron 28, 5221-5236 (1972).

16. Gibtner, T., Hampel, F., Gisselbrecht, J. P. \& Hirsch, A. End-cap stabilized oligoynes: model compounds for the linear $s p$ carbon allotrope carbyne. Chem. Eur. J. 8, 408-432 (2002).

17. Nishide, D. et al. Single-wall carbon nanotubes encaging linear chain $\mathrm{C}_{10} \mathrm{H}_{2}$ polyyne molecules inside. Chem. Phys. Lett. 428, 356-360 (2006).

18. Kitaura, R., Imazu, N., Kobayashi, K. \& Shinohara, H. Fabrication of metal nanowires in carbon nanotubes via versatile nano-template reaction. Nano Lett. 8, 693-699 (2008).

19. Chuvilin, A. et al. Self-assembly of a sulphur-terminated graphene nanoribbon within a single-walled carbon nanotube. Nature Mater. 10, 687-692 (2011).

20. Zhao, X. L., Ando, Y., Liu, Y., Jinno, M. \& Suzuki, T. Carbon nanowire made of a long linear carbon chain inserted inside a multiwalled carbon nanotube. Phys. Rev. Lett. 90, 187401 (2003).

21. Endo, M. et al. Nanotube coalescence-inducing mode: a novel vibrational mode in carbon systems. Small 2, 1031-1036 (2006).

22. Zhao, C., Kitaura, R., Hara, H., Irle, S. \& Shinohara, H. Growth of linear carbon chains inside thin double-wall carbon nanotubes. J. Phys. Chem. C 115, 13166-13170 (2011).

23. Zhang, J. et al. Synthesis and transformation of linear adamantane assemblies inside carbon nanotubes. ACS Nano 6, 8674-8683 (2012).

24. Kresse, G. \& Furthmüller, J. Efficiency of $a b$-initio total energy calculations for metals and semiconductors using a plane-wave basis set. Comp. Mater. Sci. 6, 15-50 (1996).

25. Kresse, G. \& Furthmüller, J. Efficient iterative schemes for ab initio total-energy calculations using a plane-wave basis set. Phys. Rev. B 54, 11169-11186 (1996).
26. Blöchl, P. E. Projector augmented-wave method. Phys. Rev. B 50, 17953-17979 (1994)

27. Graziano, G., Klimes, J., Fernandez-Alonso, F. \& Michaelides, A. Improved description of soft layered materials with van der Waals density functional theory. J. Phys. Condens. Matter 24, 424216 (2012).

28. Shi, L. et al. Ultra-thin double-walled carbon nanotubes: a novel nanocontainer for preparing atomic wires. Nano Res. 4, 759-766 (2011).

29. Agarwal, N. R. et al. Structure and chain polarization of long polyynes investigated with infrared and Raman spectroscopy. J. Raman Spectrosc. 44, 1398-1410 (2013).

30. Kuzmany, H. et al. Determination of SWCNT diameters from the Raman response of the radial breathing mode. Eur. Phys. J. B 22, 307-320 (2001).

31. Araujo, P. T. et al. Nature of the constant factor in the relation between radial breathing mode frequency and tube diameter for single-wall carbon nanotubes. Phys. Rev. B 77, 241403 (2008).

32. Cowley, R. A. Raman scattering from crystals of diamond structure. J. Phys.-Paris 26, 659-667 (1965).

33. Klemens, P. G. Anharmonic decay of optical phonons. Phys. Rev. 148, 845-848 (1966)

34. Balkanski, M., Wallis, R. F. \& Haro, E. Anharmonic effects in light-scattering due to optical phonons in silicon. Phys. Rev. B 28, 1928-1934 (1983).

35. Kuzmany, H. Solid-State Spectroscopy: An Introduction (Springer, 1998).

36. Kuzmany, H. Resonance Raman-scattering from neutral and doped polyacetylene. Phys. Status Solidi B 97, 521-531 (1980).

37. Yang, S. J., Kertesz, M., Zólyomi, V. \& Kürti, J. Application of a novel linear/exponential hybrid force field scaling scheme to the longitudinal Raman active mode of polyyne. J. Phys. Chem. A 111, 2434-2441 (2007).

38. Tommasini, M. et al. Intramolecular vibrational force fields for linear carbon chains through an adaptative linear scaling scheme. J. Phys. Chem. A 111, 11645-11651 (2007).

39. Lucotti, A. et al. Evidence for solution-state nonlinearity of $s p$-carbon chain based on IR and Raman spectroscopy: violation of mutual exclusion. J. Am. Chem. Soc. 131, 4239-4244 (2009).

40. Rusznyák, Zólyomi, V., Kürti, J., Yang, S. \& Kertesz, M. Bond-length alternation and charge transfer in a linear carbon chain encapsulated within a single-walled carbon nanotube. Phys. Rev. B 72, 155420 (2005).

41. Tapia, A. et al. Density functional study of the metallization of a linear carbon chain inside single wall carbon nanotubes. Carbon 48, 4057-4062 (2010).

42. Zanolli, Z., Onida, G. \& Charlier, J. C. Quantum spin transport in carbon chains. ACS Nano 4, 5174-5180 (2010).

\section{Acknowledgements}

This work was supported by the Austrian Science Fund (FWF, NanoBlends I 943-N19). L.S. acknowledges the scholarship supported by the China Scholarship Council. K.S. and Y.N. acknowledge the JST Research Acceleration Programme. J.K. and J.C.M. acknowledge financial support by the European Research Council Starting Grant No. 336453-PICOMAT. J.K. acknowledges FWF for funding through project MA14-009, as well as Vienna Scientific Cluster for computational time, and he also thanks G. Kresse and M. Marsman for their help in DFT calculations. S.C. acknowledges financial support from the Marie Curie Grant FP7-PEOPLE-2013-IEF project ID 628876.

A.R. acknowledges financial support by the European Research Council Advanced Grant DYNamo (ERC-2010-AdG-267374), POCAONTAS (FP7-PEOPLE-2012-ITN No. 316633), MINECO (FIS2013-46159-C3-1-P), Grupo Consolidado UPV/EHU del Gobierno Vasco (IT578-13), AFOSR Grant No. FA2386-15-1-0006 AOARD 144088, and COST Action MP1306 (EUSpec). Z.J.L. and L.N. acknowledge Swiss National Science Foundation (CR2212-152944). We acknowledge H. Kuzmany for his constructive discussion about the low-temperature Raman spectra of LLCCs@DWCNTs. We thank S. Puchegger from the Faculty Center for Nanostructure Research for support with SEM imaging.

\section{Author contributions}

All authors contributed to this work. L.S. and T.P. designed and supervised the experiments. L.S. prepared the samples. L.S. and P.R. did far-field Raman characterization. K.S. and Y.N. performed HRTEM characterization and simulation. J.K. and J.C.M. did the STEM measurement and simulation. J.K. did the DFT calculations. H.P. did the XRD measurement. M.W., S.C. and A.R. performed the ab initio DFT calculations. Z.J.L. and L.N. performed the near-field Raman spectroscopy of individual LLCCs@DWCNTs. L.S., P.A. and T.P. analysed data and wrote the manuscript and the Supplementary Information. All authors discussed the results and commented on the manuscript at all stages.

\section{Additional information}

Supplementary information is available in the online version of the paper. Reprints and permissions information is available online at www.nature.com/reprints. Correspondence and requests for materials should be addressed to T.P.

\section{Competing financial interests}

The authors declare no competing financial interests. 


\section{Methods}

DWCNT synthesis. DWCNTs were synthesized by HV (better than $10^{-7}$ mbar) alcohol CVD. The catalysts were obtained from the mixture of ammonium iron citrate (3 wt\%) and $\mathrm{MgO}(97 \mathrm{wt} \%)$ following ref. 43. The optimized CVD growth was performed at $875{ }^{\circ} \mathrm{C}$ in an ethanol flow of $\sim 70$ mbar for $30 \mathrm{~min}$. Typically, $0.5 \mathrm{~g}$ of catalyst powder yielded tens of milligrams of DWCNTs after two subsequent purification processes by immersion in $\mathrm{HCl}(\sim 37 \mathrm{wt} \%)$ for catalyst removal and by applying a heat treatment in air at $400^{\circ} \mathrm{C}$ for $30 \mathrm{~min}$ to remove the amorphous carbon. The resulting black powder was then immersed once again in a $\mathrm{HCl}$ solution to remove any residual catalysts. To remove chemically active SWNTs, a second annealing in air for $2 \mathrm{~h}$ was done at $500^{\circ} \mathrm{C}$ (ref. 43) and a thin DWCNT buckypaper was prepared by rinsing, filtering and drying.

Synthesis of LLCCs@DWCNTs. The LLCCs were synthesized inside DWCNTs by HTHV annealing. The pressure was lower than $8 \times 10^{-7} \mathrm{mbar}$. The optimal synthesis temperature was determined by a systematic annealing $\left(900-1,500^{\circ} \mathrm{C}\right)$ for $30 \mathrm{~min}$. To understand the growth process and to test the stability of the LLCCs, the DWCNT buckypapers were also annealed at $1,460^{\circ} \mathrm{C}$ for different time intervals (5-1,500 $\mathrm{min})$.

Sample characterization. A scanning electron microscope (SEM, Zeiss Supra $55 \mathrm{VP}$ ) was used to observe the morphology and abundance of CNTs in the as-grown sample.

Aberration-corrected HRTEM observations operated at $120 \mathrm{kV}$ (2010F, JEOL) were carried out to confirm the purity of the samples and to directly prove the structure of the LLCCs@DWCNTs. STEM (Nion UltraSTEM 100) with a medium annular dark-field detector operated at $60 \mathrm{kV}$ was also used to confirm the structure of LLCCs@DWCNTs.

The versatility of resonance Raman spectroscopy was used to study the CNTs and LLCCs using a triple-monochromator Raman spectrometer (Dilor XY with a liquid-nitrogen-cooled charge-coupled device (CCD) detector, $2 \mathrm{~mW}$ laser power, a slit width set at $200 \mu \mathrm{m}$ and a spectral resolution of about $2 \mathrm{~cm}^{-1}$ ). As excitation sources, the $568.2 \mathrm{~nm}$ line of a $\mathrm{Kr}^{+}$laser and the 587.2 and $604.3 \mathrm{~nm}$ lines of a dye laser (Rhodamine 6G) have been used. Low-temperature Raman spectra were obtained with an excitation wavelength of $568.2 \mathrm{~nm}$, combined with a cooling system (Model 22C Cryodyne Cryocooler). For comparison, all the spectra were normalized according to the intensity of the G-band.

Further near-field hyper-spectral optical imaging was used to measure the physical length of individual chains and to correlate the physical length with the Raman frequency shift. Samples were prepared by bath sonicating LLCCs@DWCNTs in 1,2-dichloroethane (Sigma-Aldrich), followed by centrifugation at $14,000 \mathrm{~g}$ for $1 \mathrm{~h}$, and then spin coating onto a glass coverslip. The measurement details can be found in the Supplementary Section 8.

Density functional theory (DFT) calculations. To improve our understanding of the optimal nanotube diameter for the growth of LLCCs, we performed density functional theory calculations with the Vienna Ab Initio simulation package (VASP; refs 24,25 ) using projector augmented wave potentials ${ }^{26}$. To properly describe the Peierls distortion, we used the HSE06 hybrid functional for exchange and correlation in our simulations ${ }^{44}$. The kinetic energy cutoff was set to $500 \mathrm{eV}$. All of the structures were placed in a $1.5 \mathrm{~nm} \times 1.5 \mathrm{~nm}$ simulation cell in the $x y$-plane, with the length in the $z$-direction defined by the equilibrium length for a two-atomic unit cell of a carbyne chain. The scheme by Monkhorst and Pack was used to generate a $\Gamma$-point centred $1 \times 1 \times 14 \mathrm{k}$-point mesh for the integration in the reciprocal space ${ }^{45}$. Van der Waals interaction was taken into account by means of the DFT-D2 method of Grimme ${ }^{46}$.

\section{References}

43. Endo, M. et al. 'Buckypaper' from coaxial nanotubes. Nature 433, 476 (2005).

44. Paier, J. et al. Screened hybrid density functionals applied to solids. J. Chem. Phys. 124, 154709 (2006).

45. Monkhorst, H. J. \& Pack, J. D. Special points for Brillouin-zone integrations. Phys. Rev. B 13, 5188-5192 (1976).

46. Grimme, S. Semiempirical GGA-type density functional constructed with a long-range dispersion correction. J. Comput. Chem. 27, 1787-1799 (2006). 